\title{
Diversidade de Syrphidae (Diptera) em cinco áreas com situações florísticas distintas no Parque Estadual Vila Velha em Ponta Grossa, Paraná1
}

\author{
Céuli M. Jorge ${ }^{2}$, Luciane Marinoni ${ }^{3} \&$ Renato C. Marinoni ${ }^{3}$
}

1. Contribuição número 1.645 do Departamento de Zoologia da Universidade Federal do Paraná.

2. Programa de Pós-graduação em Entomologia, Departamento de Zoologia, Universidade Federal do Paraná, Caixa Postal 19020, 81.531-980 Curitiba, Paraná. Bolsista da CAPES. (ceuli@ pop.com.br)

3. Departamento de Zoologia, Universidade Federal do Paraná. Bolsistas de Produtividade em Pesquisa do CNPq. (lmarinoni@ufpr.br; rcmari@ufpr.br)

\begin{abstract}
Diversity of Syrphidae (Diptera) in five areas with distinct vegetational composition in Vila Velha State Park, Ponta Grossa, Paraná, southern Brazil. The syrphids were surveyed in five floristically different areas within Vila Velha State Park in east central Paraná, Ponta Grossa, southern Brazil. Areas were classified as edge, araucaria, early (phase 1), middle (phase 2) and late (phase 3) vegetational succession, in which Malaise traps were used, with weekly collections (from September/1999 to August/2000). Nearly 300,000 Diptera were collected, in order of abundance in Araucaria area ( $\mathrm{n}=74,331$ individuals, $25 \%$ of the total), early (73,782; $25 \%)$, late $(59,339 ; 20 \%)$, middle $(53,623 ; 18 \%)$ and edge $(38,796 ; 13 \%)$. A total of 1,345 syrphids, including 97 species, were identified. The greatest abundance and richness were both observed in the edge ( $\mathrm{n}=684$ specimens in 54 species), followed by early $(\mathrm{n}=250 ; 51)$, Araucaria $(n=162 ; 34)$, late $(n=146 ; 31)$ and middle succession area $(n=103 ; 27)$. Syrphidae abundance was uncorrelated with Diptera abundance in the five areas. Syrphidae was greatest in the edge, while greatest abundance of Diptera was in the Araucaria and the least was in the edge area. Syrphinae (82\% of all Syrphidae collected), Microdontinae and Eristalinae were collected in the five areas. In the three successional stages areas (early, middle and late), Syrphinae was greatest in early, and more or less equally low in middle and late. The greatest abundance and richness occurred in most anthropogenically disturbed areas (edge and early), while the least occurred in the best preserved areas (middle and late succession). Cluster analysis showed that the two later successional stages were most similar, and the edge was the least similar to all the other areas.
\end{abstract}

KEYWORDS. Flower-flies, abundance, richness, Neotropical.

RESUMO. Um inventariamento dos sifídeos foi realizado em cinco áreas com situações florísticas diferentes dentro do Parque Estadual Vila Velha, Ponta Grossa, Paraná, sul do Brasil. As áreas foram classificadas como Borda, Araucária, estágio inicial de sucessão (Fase 1), estágio intermediário de sucessão (Fase 2) e estágio avançado de sucessão (Fase 3). As coletas foram semanais durante o período de Setembro/1999 a Agosto/2000 utilizando-se armadilha Malaise. Aproximadamente 300.000 dípteros foram capturados nas áreas (apresentadas em ordem de abundância): Araucária ( $\mathrm{n}=74.331$ indivíduos, 25\% do total), Fase 1 (73.782; 25\%), Fase 3 (59.339; 20\%), Fase $2(53.623 ; 18 \%)$ e borda $(38.796 ; 13 \%)$. Um total de 1.345 indivíduos de Syrphidae, de 97 espécies, foram identificados. As maiores abundância e riqueza de espécies foram encontradas na Borda ( $\mathrm{n}=684$ espécimes em 54 espécies), seguida pela Fase 1 (250; 51), Araucária $(162 ; 34)$, Fase $3(146 ; 31)$ e Fase $2(103 ; 27)$. A abundância de Syrphidae não se correlacionou à de Diptera em nenhuma área. Syrphidae foi mais abundante na Borda e Diptera na área de Araucária. Syrphinae ( $82 \%$ de todos os sirfídeos coletados), Microdontinae e Eristalinae foram registrados nas cinco áreas. Dos três estágios de sucessão vegetal, a subfamília Syrphinae foi mais representativa na Fase 1, ocorrendo de forma similar nas outras duas áreas. As maiores abundância e riqueza de espécies ocorreram nas áreas mais perturbadas antropicamente (Borda e Fase 1), enquanto que as menos perturbadas apresentaram menores abundância e riqueza (Fase 2 e Fase 3). A análise de agrupamento mostrou que as áreas em sucessão vegetal avançadas são mais similares e a de borda a mais diferenciada.

PALAVRAS-CHAVE. Moscas-das-flores, abundância, riqueza de espécies, Neotropical.

Muitos trabalhos têm sido realizados utilizando os insetos como subsídios para estudos de avaliação de condições ambientais (SouthwOOD et al., 1979; LAWTON, 1983; Humphrey et al., 1998; GANHO \& Marinoni, 2003; MARINONi \& GANHo, 2003). Isso se deve ao fato dos insetos serem facilmente atraídos e capturados, ocuparem os mais diversos habitats, possuírem uma grande biomassa e atuarem em vários níveis tróficos.

Por conseguinte, partindo do reconhecimento da importância dos insetos como potenciais indicadores ambientais, dois grandes inventariamentos foram realizados no Estado do Paraná: o Projeto de Levantamento da Fauna Entomológica do Paraná - PROFAUPAR e o Projeto Vila Velha-PROVIVE. O PROFAUPAR foi realizado no período de agosto de 1986 a setembro de 1988, em oito regiões fitogeográficas diferentes no Paraná, dando início a um processo de inventariamentos de Insecta no Estado
(MARINONI \& DUTRA, 1993). O PROVIVE foi realizado, por três anos consecutivos, no período de 1999 a 2002, em Vila Velha, Ponta Grossa. Esse projeto teve como objetivo caracterizar a fauna entomológica em cinco áreas com diferentes condições de preservação e alteração antrópica, utilizando-se armadilhas Malaise e de solo (GANho \& Marinoni, 2003; Marinoni \& Ganho, 2003). MARINONI et al. (2004) analisaram a abundância e a riqueza de espécies de Syrphidae em duas das áreas amostradas durante o primeiro ano do projeto: a área de Borda e a área do interior da floresta (Fase 2) nesta última realizaramse coletas durante o PROFAUPAR, dezessete anos antes. No presente trabalho, a fauna de Syrphidae é avaliada nas cinco áreas amostradas durante o primeiro ano do PROVIVE (1999/2000), buscando contribuir para o conhecimento da família no Estado do Paraná (e conseqüentemente, na região Neotropical). 


\section{MATERIALE MÉTODOS}

Foram selecionadas cinco áreas com diferentes estágios de sucessão vegetal ou em diferentes condições ambientais por manejo no Parque Estadual Vila Velha, localizado no município de Ponta Grossa, junto à Rodovia do Café, BR 376, km 83. O Parque está situado entre as coordenadas $25^{\circ} 12^{\prime} 34^{\prime \prime}$ e $25^{\circ} 15^{\prime} 35^{\prime \prime}$ de latitude Sul e $49^{\circ} 58^{\prime}$ 04" e 50 03 '37" de longitude Oeste, a 880 metros de altitude e possui área de $5.032 .384 \mathrm{~m}^{2}$.

As cinco áreas foram denominadas: Borda, área de Araucária, Fase 1, Fase 2 e Fase 3 de sucessão. A Borda caracteriza-se como área de transição entre vegetação arbórea em estágio intermediário de sucessão e área de campo. A área de Araucária é um plantio de Araucaria angustifolia (Araucariaceae), cujo manejo de limpeza deixou de ser feito há mais de 20 anos. As três áreas restantes representam, segundo os pesquisadores $\mathrm{F}$. Galvão e Y.S. Kuniyoshi que realizaram a caracterização das áreas (GANHO \& MARINONI, 2003), uma sequiência no processo de sucessão da Floresta Ombrófila Mista. A Fase 1, em estágio inicial de sucessão vegetal, foi anteriormente utilizada para culturas agrícolas sazonais, (milho, feijão) e encontra-se em estágio de recuperação há 20 anos. A Fase 2, em estágio intermediário de sucessão, é caracterizada como floresta primária alterada pela retirada de várias essências vegetais. A Fase 3, mais conservada, (estado avançado de sucessão), ainda apresentando traços de floresta primária, foi alterada por cortes seletivos.

Em cada área foi instalada uma armadilha Malaise com as modificações descritas em MARINONI \& DUTRA (1993). O material foi recolhido semanalmente, no período de 06 de setembro de 1999 a 28 de agosto de 2000, totalizando 52 amostras/área.

Mais detalhes sobre os locais e a metodologia de coleta podem ser encontrados em GANHO \& MARINONI (2003), MARINONI \& GANHO (2003) e MARINONI et al. (2004).

A identificação dos sirfídeos foi realizada com bibliografia pertinente à família (HARBACH, 1984; HIPPA \& Thompson, 1983; Thompson, 1969, 1981a, 1981b, 1991, 1997a, 1997b, 1999), por comparação com material já identificado na Coleção Entomológica Pe. Jesus Santiago Moure (DZUP) do Departamento de Zoologia da Universidade Federal do Paraná e com o auxílio de F. Christian Thompson, do National Museum of Natural History, Smithsonian Institution, Washington, D.C., E.U.A.

O material está depositado na Coleção Entomológica Pe. Jesus S. Moure e todos os dados referentes aos espécimes coletados estão registrados no programa gerenciador de biodiversidade, BONABio, sob a plataforma MS Access, tendo sido a interface e a elaboração da base de dados realizadas por Sionei Ricardo Bonatto. A informação dos espécimes pode ser acessada via internet por meio do projeto Taxon line - Rede Paranaense de Coleções Biológicas (www.taxonline.ufpr.br).

A abundância e a riqueza de espécies de Syrphidae foram descritas e comparadas entre as cinco áreas inventariadas, bem como a abundância de Diptera. Para análise de riqueza e confecção de curva de acumulação de espécies foram utilizados os dados semanais de cada coleta.
A comparação das áreas com base em presença de espécies capturadas foi realizada através de análise de agrupamento utilizando-se o programa NTSYS-pc 1.80 (RoHLF, 1989). Para construção do fenograma foi utilizado o coeficiente de semelhança de Jaccard e agrupamento por UPGMA (SNEATH \& SOKAL, 1973).

Para se estabelecer a guilda trófica dos sirfídeos tem-se como base o hábito alimentar dos imaturos, uma vez que na fase adulta todos se alimentam de néctar e/ou pólen. Para avaliação das guildas tróficas de Syrphidae, utilizou-se a classificação proposta por GILBERT (1985).

Os estimadores de riqueza de espécies foram calculados com o programa Richness EstimateS 6.0b 1 (Cowell, 2000) pelos métodos Chao 1, Chao 2, Jack-Knife 1, Jack-Knife 2, Bootstrap e Michaelis-Menten.

\section{RESULTADOS}

Abundância de Diptera. Foram capturados 299.871 dípteros nas cinco áreas amostradas. A área com maior abundância foi a de Araucária, com 74.331 exemplares (25\%); seguida da Fase 1, com 73.782 exemplares (24\%); Fase 3, com 59.339 (20\%); Fase 2, com 53.623 (18\%) e da Borda, com 38.796 dípteros (13\%).

Abundância e riqueza de espécies de Syrphidae. Foram coletados 1.345 exemplares de Syrphidae nas cinco áreas, sendo reconhecidas 97 espécies em 26 gêneros (Tabs. I-III). Aárea com maior abundância e riqueza foi a de Borda, com 684 exemplares capturados $(51 \%)$ de 54 espécies; seguida pela Fase 1, com 250 (19\%) de 51; Araucária, com 162 (12\%) de 34; Fase 3, com 146 (10\%) de 31 e Fase 2, com 103 (8\%) de 27.

A abundância de exemplares de Syrphidae nas cinco áreas foi inversamente proporcional à de exemplares de Diptera, sendo isso mais evidente na Borda, onde ocorreu a maior captura de Syrphidae e a menor de Diptera.

A ordem de riqueza de espécies acompanhou a ordem de abundância nas cinco áreas. As maiores riqueza e abundância foram encontradas na Borda. As áreas com estágio de sucessão vegetal menos avançado (Fase 1) e área de Araucária apresentaram maiores riqueza e abundância do que as áreas mais preservadas (Fase $2 \mathrm{e}$ Fase 3).

As três subfamílias de Syrphidae (Syrphinae, Microdontinae e Eristalinae) foram registradas nas cinco áreas, sendo que a maioria dos exemplares $(82 \%)$ foi de Syrphinae (Tab. IV).

A seguir são apresentados os resultados em cada uma das áreas por ordem decrescente de abundância.

Área de Borda. Na Borda foram coletados 684 indivíduos (14 exemplares/coleta), os quais foram identificados em 54 espécies. Vinte e oito espécies foram exclusivas (Tabs. I-III). A espécie em maior abundância foi Allograpta neotropica Curran, 1936 (150 exemplares); a segunda mais abundante foi Syrphus phaeostigma Wiedemann, 1830 (134) e a terceira Toxomerus procrastinatus Metz, 2001 (59). A soma dos totais capturados dessas três espécies representa o equivalente a 50\% da captura de Syrphinae nessa área. Foram reconhecidos 17 gêneros (Tab. V). Os gêneros com maior riqueza foram Ocyptamus Macquart, 1834 (17 espécies) e 
Tabela I. Abundância e riqueza de Syrphinae (Syrphidae) nas cinco áreas inventariadas do Parque Estadual Vila Velha, Ponta Grossa, Paraná, no período de setembro de 1999 a agosto de 2000 (M, machos; F, fêmeas; * espécies exclusivas).

\begin{tabular}{|c|c|c|c|c|c|c|c|c|c|c|c|}
\hline \multirow[t]{2}{*}{ Syrphinae } & \multicolumn{2}{|c|}{ Borda } & \multicolumn{2}{|c|}{ Araucária } & \multicolumn{2}{|c|}{ Fase 1} & \multicolumn{2}{|c|}{ Fase 2} & \multicolumn{2}{|c|}{ Fase 3} & \multirow[t]{2}{*}{ Total } \\
\hline & $\mathrm{M}$ & $\mathrm{F}$ & M & $\mathrm{F}$ & $\mathrm{M}$ & $\mathrm{F}$ & $\mathrm{M}$ & $\mathrm{F}$ & $\mathrm{M}$ & $\mathrm{F}$ & \\
\hline Allograpta exotica (Wiedemann, 1830) & 14 & 18 & 0 & 1 & 2 & 1 & 0 & 0 & 1 & 1 & 38 \\
\hline Allograpta neotropica Curran, 1936 & 73 & 77 & 1 & 0 & 4 & 5 & 1 & 1 & 0 & 0 & 162 \\
\hline Allograpta similis Curran, $1925^{*}$ & 0 & 2 & 0 & 0 & 0 & 0 & 0 & 0 & 0 & 0 & 2 \\
\hline Allograpta sp. $1^{*}$ & 0 & 1 & 0 & 0 & 0 & 0 & 0 & 0 & 0 & 0 & 1 \\
\hline Allograpta sp. $2 *$ & 0 & 1 & 0 & 0 & 0 & 0 & 0 & 0 & 0 & 0 & 1 \\
\hline Argentinomyia pollinosa (Hull, 1942)* & 0 & 0 & 0 & 0 & 0 & 1 & 0 & 0 & 0 & 0 & 1 \\
\hline Argentinomyia sp. $1^{*}$ & 0 & 1 & 0 & 0 & 0 & 0 & 0 & 0 & 0 & 0 & 1 \\
\hline Argentinomyia sp. $2 *$ & 0 & 0 & 0 & 0 & 1 & 0 & 0 & 0 & 0 & 0 & 1 \\
\hline Leucopodella balboa (Hull, 1947)* & 0 & 0 & 0 & 1 & 0 & 0 & 0 & 0 & 0 & 0 & 1 \\
\hline Leucopodella gracilis (Williston, 1891) & 1 & 6 & 0 & 6 & 0 & 3 & 0 & 2 & 0 & 10 & 28 \\
\hline Ocyptamus alicia (Curran, 1941)* & 1 & 3 & 0 & 0 & 0 & 0 & 0 & 0 & 0 & 0 & 4 \\
\hline Ocyptamus antiphates (Walker, 1849)* & 1 & 8 & 0 & 0 & 0 & 0 & 0 & 0 & 0 & 0 & 9 \\
\hline Ocyptamus aster (Curran, 1941)* & 1 & 0 & 0 & 0 & 0 & 0 & 0 & 0 & 0 & 0 & 1 \\
\hline Ocyptamus bonariensis (Brethes, 1905) & 1 & 0 & 0 & 0 & 0 & 1 & 0 & 0 & 1 & 1 & 4 \\
\hline Ocyptamus clarapex (Wiedemann, 1830)* & 0 & 1 & 0 & 0 & 0 & 0 & 0 & 0 & 0 & 0 & 1 \\
\hline Ocyptamus erebus (Hull, 1943) & 1 & 2 & 0 & 2 & 0 & 3 & 0 & 0 & 0 & 0 & 8 \\
\hline Ocyptamus funebris Macquart, 1834 & 2 & 21 & 1 & 9 & 0 & 10 & 0 & 1 & 0 & 4 & 48 \\
\hline Ocyptamus gastrostactus (Wiedemann, 1830) & 0 & 13 & 0 & 0 & 0 & 1 & 0 & 0 & 0 & 0 & 14 \\
\hline Ocyptamus hyalipennis (Curran, 1930) & 1 & 0 & 0 & 1 & 0 & 2 & 0 & 0 & 0 & 0 & 4 \\
\hline Ocyptamus lividus (Schiner, 1868)* & 0 & 2 & 0 & 0 & 0 & 0 & 0 & 0 & 0 & 0 & 2 \\
\hline Ocyptamus rugosifrons (Schiner, 1868)* & 1 & 0 & 0 & 0 & 0 & 0 & 0 & 0 & 0 & 0 & 1 \\
\hline Ocyptamus sativus (Curran, 1941) & 0 & 0 & 1 & 7 & 2 & 11 & 2 & 12 & 0 & 11 & 46 \\
\hline Ocyptamus stenogaster (Williston, 1888)* & 2 & 2 & 0 & 0 & 0 & 0 & 0 & 0 & 0 & 0 & 4 \\
\hline Ocyptamus sp. 1 & 0 & 0 & 0 & 0 & 1 & 5 & 1 & 12 & 0 & 9 & 28 \\
\hline Ocyptamus sp. 2 & 0 & 0 & 0 & 3 & 0 & 0 & 0 & 2 & 0 & 1 & 6 \\
\hline Ocyptamus sp. $3 *$ & 0 & 0 & 0 & 0 & 0 & 1 & 0 & 0 & 0 & 0 & 1 \\
\hline Ocyptamus sp. 4 & 0 & 0 & 0 & 0 & 0 & 8 & 0 & 0 & 0 & 2 & 10 \\
\hline Ocyptamus sp. $5 *$ & 0 & 0 & 0 & 0 & 0 & 0 & 1 & 0 & 0 & 0 & 1 \\
\hline Ocyptamus sp. 6 & 0 & 1 & 0 & 0 & 0 & 2 & 1 & 0 & 0 & 0 & 4 \\
\hline Ocyptamus sp. 7* & 0 & 0 & 0 & 2 & 0 & 0 & 0 & 0 & 0 & 0 & 2 \\
\hline Ocyptamus sp. 8* & 0 & 1 & 0 & 0 & 0 & 0 & 0 & 0 & 0 & 0 & 1 \\
\hline Ocyptamus sp. 9* & 0 & 1 & 0 & 0 & 0 & 0 & 0 & 0 & 0 & 0 & 1 \\
\hline Ocyptamus sp. $10 *$ & 2 & 12 & 0 & 0 & 0 & 0 & 0 & 0 & 0 & 0 & 14 \\
\hline Ocyptamus sp. 11 & 0 & 2 & 0 & 0 & 0 & 3 & 1 & 0 & 0 & 0 & 6 \\
\hline Pseudodoros clavatus (Fabricius, 1794) & 8 & 12 & 0 & 0 & 0 & 1 & 0 & 0 & 0 & 0 & 21 \\
\hline Syrphus phaeostigma Wiedemann, 1830 & 72 & 62 & 18 & 35 & 22 & 39 & 0 & 1 & 2 & 6 & 257 \\
\hline Toxomerus confusus (Schiner, 1868) & 2 & 4 & 2 & 4 & 5 & 7 & 0 & 0 & 0 & 0 & 24 \\
\hline Toxomerus croesus (Hull, 1940) & 0 & 0 & 2 & 1 & 4 & 4 & 6 & 4 & 12 & 7 & 40 \\
\hline Toxomerus dispar (Fabricius, 1794)* & 0 & 2 & 0 & 0 & 0 & 0 & 0 & 0 & 0 & 0 & 2 \\
\hline Toxomerus musicus (Fabricius, 1805) & 39 & 12 & 0 & 0 & 0 & 0 & 0 & 0 & 0 & 0 & 51 \\
\hline Toxomerus nitidiventris (Curran 1930) & 0 & 3 & 0 & 0 & 3 & 0 & 0 & 0 & 0 & 0 & 6 \\
\hline Toxomerus pictus (Macquart, 1842)* & 4 & 1 & 0 & 0 & 0 & 0 & 0 & 0 & 0 & 0 & 5 \\
\hline Toxomerus politus (Say, 1823) & 27 & 23 & 0 & 1 & 0 & 2 & 0 & 0 & 0 & 0 & 53 \\
\hline Toxomerus procrastinatus Metz, 2001 & 32 & 27 & 6 & 1 & 19 & 11 & 2 & 0 & 18 & 1 & 117 \\
\hline Toxomerus tibicen (Wiedemann, 1830) & 32 & 15 & 4 & 0 & 6 & 1 & 0 & 1 & 2 & 1 & 62 \\
\hline Toxomerus sp. $1^{*}$ & 0 & 1 & 0 & 0 & 0 & 0 & 0 & 0 & 0 & 0 & 1 \\
\hline Toxomerus sp. $2 *$ & 0 & 2 & 0 & 0 & 0 & 0 & 0 & 0 & 0 & 0 & 2 \\
\hline Toxomerus sp. $3 *$ & 0 & 1 & 0 & 0 & 0 & 0 & 0 & 0 & 0 & 0 & 1 \\
\hline Toxomerus sp. 4* & 0 & 1 & 0 & 0 & 0 & 0 & 0 & 0 & 0 & 0 & 1 \\
\hline Toxomerus sp. 5* & 0 & 2 & 0 & 0 & 0 & 0 & 0 & 0 & 0 & 0 & 2 \\
\hline Toxomerus sp. 6* & 2 & 1 & 0 & 0 & 0 & 0 & 0 & 0 & 0 & 0 & 3 \\
\hline Total de exemplares & 319 & 344 & 35 & 74 & 69 & 122 & 15 & 36 & 36 & 54 & 1.104 \\
\hline Total de espécies (51) & \multicolumn{2}{|c|}{40} & \multicolumn{2}{|c|}{16} & \multicolumn{2}{|c|}{24} & \multicolumn{2}{|c|}{13} & \multicolumn{2}{|c|}{12} & \\
\hline
\end{tabular}

Toxomerus Macquart, 1855 (14), sendo que os demais apresentaram de uma a cinco espécies. A maior abundância foi apresentada por Toxomerus e a segunda maior por Allograpta Osten Sacken, 1875.

A maioria dos exemplares capturados nesta área foi da subfamília Syrphinae (97\%), seguida por Eristalinae (2\%) e Microdontinae (1\%) (Tab. IV).

Quando comparada às demais áreas, a Borda foi a que apresentou maior abundância e riqueza de espécies, tendo praticamente a metade do número total de exemplares capturados no conjunto das cinco áreas.

Até a $49^{a}$ coleta, novas espécies ainda estavam sendo acrescentadas, não havendo estabilização da curva de acumulação de espécies. Isso indica que, possivelmente, outras espécies podem ocorrer nesse local, o que poderá ser demonstrado com a análise do material do segundo e terceiro anos do projeto.

Área Fase 1. Nesta área, foram capturados 250 indivíduos (cinco exemplares/coleta), os quais foram identificados em 51 espécies. Dentre as espécies, 11 foram registradas exclusivamente neste local (Tabs. I-III). A espécie mais abundante foi Syrphus phaeostigma (61 exemplares); seguida de $T$. procrastinatus (30) e Ocyptamus sativus (Curran, 1941) (13).

Foram identificados 20 gêneros, sendo esta, portanto, a área com maior número de gêneros entre as 
Tabela II. Abundância e riqueza de Microdontinae (Syrphidae) nas cinco áreas inventariadas do Parque Estadual Vila Velha, Ponta Grossa, Paraná, no período de setembro de 1999 a agosto de 2000 (M, machos; F, fêmeas; * espécies exclusivas).

\begin{tabular}{|c|c|c|c|c|c|c|c|c|c|c|c|}
\hline \multirow[t]{2}{*}{ Microdontinae } & \multicolumn{2}{|c|}{ Borda } & \multicolumn{2}{|c|}{ Araucária } & \multicolumn{2}{|c|}{ Fase 1} & \multicolumn{2}{|c|}{ Fase 2} & \multicolumn{2}{|c|}{ Fase 3} & \multirow[t]{2}{*}{ Total } \\
\hline & M & $\mathrm{F}$ & M & $\mathrm{F}$ & M & $\mathrm{F}$ & M & $\mathrm{F}$ & M & $\mathrm{F}$ & \\
\hline Aristosyrphus sp. 1 & 0 & 0 & 0 & 1 & 0 & 1 & 0 & 0 & 0 & 1 & 3 \\
\hline Aristosyrphus minutus Thompson, 2004 & 0 & 0 & 0 & 3 & 0 & 3 & 0 & 4 & 0 & 0 & 10 \\
\hline Aristosyrphus sp. 2 & 0 & 0 & 0 & 1 & 0 & 3 & 0 & 0 & 0 & 0 & 4 \\
\hline Microdon aureopilis Marinoni, 2004 & 0 & 0 & 5 & 3 & 0 & 2 & 0 & 3 & 0 & 1 & 14 \\
\hline Microdon aurifex Wiedemann, 1830 & 0 & 0 & 3 & 0 & 0 & 1 & 6 & 1 & 3 & 2 & 16 \\
\hline Microdon mitis Curran, 1940 & 0 & 1 & 0 & 0 & 0 & 0 & 0 & 0 & 7 & 3 & 11 \\
\hline Microdon mourei Thompson, 2004 & 0 & 0 & 0 & 0 & 1 & 0 & 1 & 0 & 2 & 0 & 4 \\
\hline Microdon nero Curran, 1936 & 0 & 1 & 0 & 3 & 0 & 1 & 0 & 0 & 0 & 0 & 5 \\
\hline Microdon pilosops Marinoni, 2004 & 0 & 0 & 0 & 0 & 0 & 1 & 0 & 0 & 1 & 0 & 2 \\
\hline Microdon sepulchrasilvus (Hull, 1937) & 0 & 1 & 0 & 1 & 0 & 0 & 0 & 0 & 0 & 0 & 2 \\
\hline Microdon tigrinus Curran, 1940 & 0 & 0 & 1 & 2 & 6 & 1 & 3 & 1 & 0 & 1 & 15 \\
\hline Microdon virgo Curran, 1940 & 0 & 0 & 1 & 1 & 0 & 0 & 1 & 1 & 1 & 9 & 14 \\
\hline Microdon sp. $1 *$ & 0 & 0 & 0 & 1 & 0 & 0 & 0 & 0 & 0 & 0 & 1 \\
\hline Microdon sp. $2 *$ & 0 & 0 & 0 & 0 & 1 & 0 & 0 & 0 & 0 & 0 & 1 \\
\hline Mixogaster polistes Hull, 1954 & 0 & 1 & 1 & 10 & 5 & 4 & 1 & 4 & 0 & 1 & 27 \\
\hline Mixogaster sartocryptus Hull, 1954 * & 0 & 0 & 0 & 0 & 0 & 0 & 0 & 0 & 8 & 3 & 11 \\
\hline Paramicrodon flukei (Curran, 1936) & 0 & 0 & 1 & 3 & 0 & 2 & 0 & 0 & 0 & 0 & 6 \\
\hline Paramicrodon sp. 1 & 0 & 0 & 0 & 1 & 0 & 0 & 0 & 0 & 1 & 0 & 2 \\
\hline Paramicrodon sp. 2 & 0 & 1 & 0 & 0 & 1 & 0 & 0 & 0 & 0 & 0 & 2 \\
\hline Total de exemplares & 0 & 5 & 12 & 30 & 14 & 19 & 12 & 14 & 23 & 21 & 150 \\
\hline Total de espécies (19) & \multicolumn{2}{|c|}{5} & \multicolumn{2}{|c|}{13} & \multicolumn{2}{|c|}{13} & \multicolumn{2}{|c|}{7} & \multicolumn{2}{|c|}{11} & \\
\hline
\end{tabular}

Tabela III. Abundância e riqueza de Eristalinae (Syrphidae) nas cinco áreas inventariadas do Parque Estadual Vila Velha, Ponta Grossa, Paraná, no período de setembro de 1999 a agosto de 2000. (M, machos; F, fêmeas; * espécies exclusivas).

\begin{tabular}{|c|c|c|c|c|c|c|c|c|c|c|c|}
\hline \multirow[t]{2}{*}{ Eristalinae } & \multicolumn{2}{|c|}{ Borda } & \multicolumn{2}{|c|}{ Araucária } & \multicolumn{2}{|c|}{ Fase 1} & \multicolumn{2}{|c|}{ Fase 2} & \multicolumn{2}{|c|}{ Fase 3} & \multirow[t]{2}{*}{ Total } \\
\hline & $\mathrm{M}$ & $\mathrm{F}$ & $\mathrm{M}$ & $\mathrm{F}$ & M & $\mathrm{F}$ & $\mathrm{M}$ & $\mathrm{F}$ & $\mathrm{M}$ & $\mathrm{F}$ & \\
\hline Ceriogaster sp. $1 *$ & 0 & 3 & 0 & 0 & 0 & 0 & 0 & 0 & 0 & 0 & 3 \\
\hline Copestylum albifrons (Curran, 1939)* & 0 & 0 & 0 & 0 & 0 & 1 & 0 & 0 & 0 & 0 & 1 \\
\hline Copestylum circe (Curran, 1939)* & 0 & 0 & 0 & 0 & 0 & 0 & 0 & 0 & 0 & 2 & 2 \\
\hline Copestylum circumdatum (Walker 1857)* & 0 & 0 & 0 & 0 & 1 & 0 & 0 & 0 & 0 & 0 & 1 \\
\hline Copestylum flukei (Curran, 1936)* & 0 & 0 & 0 & 0 & 0 & 0 & 0 & 1 & 0 & 0 & 1 \\
\hline Copestylum macquarti (Curran,1926) & 0 & 0 & 0 & 2 & 0 & 3 & 0 & 9 & 0 & 1 & 15 \\
\hline Copestylum pictum (Wiedemann, 1830) & 0 & 0 & 0 & 0 & 0 & 1 & 0 & 2 & 0 & 0 & 3 \\
\hline Copestylum sp. $1^{*}$ & 2 & 2 & 0 & 0 & 0 & 0 & 0 & 0 & 0 & 0 & 4 \\
\hline Copestylum sp. 2 & 0 & 1 & 0 & 3 & 0 & 0 & 0 & 0 & 0 & 0 & 4 \\
\hline Copestylum sp. $3 *$ & 0 & 0 & 0 & 0 & 0 & 0 & 0 & 0 & 0 & 1 & 1 \\
\hline Copestylum selectum (Curran, 1939) & 0 & 1 & 0 & 2 & 0 & 2 & 1 & 6 & 0 & 1 & 13 \\
\hline Eumerus obliquus (Fabricius 1805)* & 0 & 0 & 0 & 0 & 1 & 0 & 0 & 0 & 0 & 0 & 1 \\
\hline Meromacrus nectarinoides (Lynch-Arribálzaga, 1892)* & 0 & 0 & 0 & 0 & 0 & 1 & 0 & 0 & 0 & 0 & 1 \\
\hline Myolepta sp. $1^{*}$ & 0 & 0 & 0 & 0 & 1 & 0 & 0 & 0 & 0 & 0 & 1 \\
\hline Nausigaster tuberculata (Carrera, Lopes \& Lane, 1947)* & 0 & 1 & 0 & 0 & 0 & 0 & 0 & 0 & 0 & 0 & 1 \\
\hline Neplas armatipes (Curran, 1941) & 0 & 0 & 1 & 2 & 5 & 4 & 0 & 4 & 2 & 1 & 19 \\
\hline Neplas sp. 1 & 1 & 0 & 0 & 0 & 0 & 1 & 0 & 0 & 0 & 0 & 2 \\
\hline Palpada sp. $1^{*}$ & 0 & 0 & 0 & 0 & 0 & 0 & 0 & 0 & 0 & 1 & 1 \\
\hline Ornidia obesa (Fabricius, 1775) & 3 & 0 & 0 & 0 & 1 & 1 & 0 & 0 & 0 & 0 & 5 \\
\hline Polybiomya wulpii (Williston 1888)* & 0 & 0 & 0 & 0 & 0 & 1 & 0 & 0 & 0 & 0 & 1 \\
\hline Quichuana bezzi Ceresa, 1934* & 1 & 0 & 0 & 0 & 0 & 0 & 0 & 0 & 0 & 0 & 1 \\
\hline Spilomyia gratiosa Wulp, 1888* & 0 & 0 & 0 & 0 & 1 & 0 & 0 & 0 & 0 & 0 & 1 \\
\hline Sterphus sp. $1 *$ & 0 & 0 & 0 & 1 & 0 & 0 & 0 & 0 & 0 & 0 & 1 \\
\hline Sphiximorpha acra (Curran 1941) & 0 & 0 & 0 & 0 & 1 & 0 & 0 & 0 & 0 & 2 & 3 \\
\hline Sphiximorpha barbipes (Loew 1853)* & 0 & 0 & 0 & 0 & 0 & 0 & 0 & 2 & 0 & 0 & 2 \\
\hline Sphiximorpha sp. 1 & 0 & 0 & 0 & 0 & 0 & 0 & 0 & 1 & 0 & 1 & 2 \\
\hline Trichopsomya sp. $1 *$ & 0 & 1 & 0 & 0 & 0 & 0 & 0 & 0 & 0 & 0 & 1 \\
\hline Total de exemplares & 7 & 9 & 1 & 10 & 11 & 15 & 1 & 25 & 2 & 10 & 91 \\
\hline Total de espécies (27) & \multicolumn{2}{|c|}{9} & \multicolumn{2}{|c|}{5} & \multicolumn{2}{|c|}{14} & \multicolumn{2}{|c|}{7} & \multicolumn{2}{|c|}{8} & \\
\hline
\end{tabular}

cinco inventariadas (Tab. V). Os gêneros com maior riqueza foram Ocyptamus (11 espécies); Microdon Meigen, 1803 (7) e Toxomerus (6).

Da mesma forma que na Borda, a maioria dos exemplares coletados pertence a Syrphinae $(76 \%)$, seguida por Microdontinae (14\%) e Eristalinae (10\%).

Na $27^{a}$ semana de coletas, $92 \%$ das espécies (51) já haviam sido capturadas. A assíntota foi alcançada na $43^{\mathrm{a}}$ semana.

Área de Araucária. Nesta área foram coletados 162 indivíduos (três exemplares/coleta) de 34 espécies. Dentre as espécies capturadas, somente quatro foram exclusivas (Tabs. I-III). Da mesma forma que na Fase 1, a espécie mais abundante foi S. phaeostigma, com 53 exemplares; a segunda mais abundante foi Mixogaster polistes Hull, 1954 (11) e a terceira Ocyptamus funebris Macquart, 1834 (10). A captura de S. phaeostigma representou 33\% do total capturado na área.

Foram reconhecidos 12 gêneros (Tab. V). O mais abundante foi Syrphus (53 espécimes) e os que 
apresentaram maior riqueza foram: Microdon (7); Ocyptamus (6) e Toxomerus (5). Nessa área, a maioria dos exemplares também foi de Syrphinae $(67 \%)$, seguida por Microdontinae (26\%) e Eristalinae (7\%).

Até a $22^{\mathrm{a}}$ semana de coleta, ocorreu uma rápida ascendência da curva de acumulação de espécies e $82 \%$ das mesmas (28) haviam sido coletadas. A partir da $22^{\mathrm{a}}$ coleta, a curva começou a estabilizar, aumentando, em média, uma espécie a cada quatro coletas até a $40^{\mathrm{a}}$ coleta.

Área Fase 3. Foram capturados 146 indivíduos (três exemplares/coleta) os quais foram identificados em 31 espécies (Tabs. I-III). Do total de espécies, quatro foram registradas exclusivamente nessa área. As espécies mais abundantes foram Toxomerus croesus (Hull, 1940) e $T$. procrastinatus, com 19 espécimes cada; seguidas por $O$. sativus e Mixogaster sartocryptus Hull, 1954, com 11; Leucopodella gracilis (Williston, 1891), Microdon mitis Curran, 1940 e Microdon virgo Curran, 1940, com 10.

Treze gêneros foram reconhecidos (Tab. V). Os mais ricos foram Microdon, com sete espécies, e Ocyptamus, com seis. A maioria dos exemplares foi de Syrphinae (62\%), seguida por Microdontinae (30\%) e Eristalinae $(8 \%)$. Dentre as cinco áreas, esta foi a que apresentou maior número de representantes de Microdontinae, embora Syrphinae tenha sido a mais abundante.
$\mathrm{Na} 20^{a}$ semana de coletas, $94 \%$ das espécies (29) já haviam sido capturadas, ocorrendo uma estabilização da curva de acumulação de espécies.

Área Fase 2. Foram capturados 103 indivíduos nessa área (dois exemplares/coleta), os quais foram identificados em 27 espécies. Do total, somente três foram exclusivas (Tabs. I-III). A espécie mais abundante foi $O$. sativus, com 14 exemplares; seguida de Ocyptamus sp. 1 (13) e $T$. croesus (10).

Onze gêneros foram reconhecidos (Tab. V). Os com maior riqueza foram Ocyptamus (7) e Microdon (5). A maioria dos exemplares pertence a Syrphinae (50\%), seguida por Microdontinae (25\%) e Eristalinae (25\%).

Na $19^{a}$ semana de coletas, $89 \%$ das espécies (24) já haviam sido registradas, ocorrendo uma estabilização da curva de acumulação na $49^{\mathrm{a}}$ semana.

Gêneros e espécies de Syrphidae capturados em maior riqueza e abundância. A subfamília que apresentou maior riqueza e abundância foi Syrphinae, com 51 espécies identificadas em 1.104 exemplares coletados (Tabs. I, IV). Os exemplares de Syrphinae representaram 82\% do total de Syrphidae capturado nas cinco áreas.

Do total, foram registradas 40 espécies em 663 indivíduos na Borda; 16 espécies em 109 indivíduos na área de Araucária; 24 espécies em 191 indivíduos na Fase

Tabela IV. Abundância e riqueza de Syrphinae, Microdontinae e Eristalinae (Syrphidae) nas cinco áreas inventariadas do Parque Estadual Vila Velha, Ponta Grossa, Paraná, no período de setembro de 1999 a agosto de 2000 (M, machos; F, fêmeas).

\begin{tabular}{|c|c|c|c|c|c|c|c|c|c|c|c|}
\hline \multirow[t]{2}{*}{ Subfamílias } & \multicolumn{2}{|c|}{ Borda } & \multicolumn{2}{|c|}{ Araucária } & \multicolumn{2}{|c|}{ Fase 1} & \multicolumn{2}{|c|}{ Fase 2} & \multicolumn{2}{|c|}{ Fase 3} & \multirow[t]{2}{*}{ Total } \\
\hline & $\mathrm{M}$ & $\mathrm{F}$ & $\mathrm{M}$ & $\mathrm{F}$ & $\mathrm{M}$ & $\mathrm{F}$ & $\mathrm{M}$ & $\mathrm{F}$ & $\mathrm{M}$ & $\mathrm{F}$ & \\
\hline Syrphinae & 319 & 344 & 35 & 74 & 69 & 122 & 15 & 36 & 36 & 54 & 1104 \\
\hline Eristalinae & 7 & 9 & 1 & 10 & 11 & 15 & 1 & 25 & 2 & 10 & 91 \\
\hline Microdontinae & 0 & 5 & 12 & 30 & 14 & 19 & 12 & 14 & 23 & 21 & 150 \\
\hline Total de exemplares & 326 & 358 & 48 & 114 & 94 & 156 & 28 & 75 & 61 & 85 & 1.345 \\
\hline Total de Espécies (97) & \multicolumn{2}{|c|}{54} & \multicolumn{2}{|c|}{34} & \multicolumn{2}{|c|}{51} & \multicolumn{2}{|c|}{27} & \multicolumn{2}{|c|}{31} & \\
\hline
\end{tabular}

Tabela V. Abundância ( ${ }^{\circ}$ de indivíduos) e total de espécies dos gêneros de Syrphidae registrados nas cinco áreas inventariadas do Parque Estadual Vila Velha, Ponta Grossa, Paraná, no período de setembro de 1999 a agosto de 2000.

\begin{tabular}{|c|c|c|c|c|c|c|c|c|c|c|c|c|}
\hline \multirow[t]{2}{*}{ Gênero } & \multicolumn{2}{|c|}{ Borda } & \multicolumn{2}{|c|}{ Araucária } & \multicolumn{2}{|c|}{ Fase 1} & \multicolumn{2}{|c|}{ Fase 2} & \multicolumn{2}{|c|}{ Fase 3} & \multirow[t]{2}{*}{ Total } & \multirow[t]{2}{*}{ Espécies } \\
\hline & $\mathrm{M}$ & $\mathrm{F}$ & $\mathrm{M}$ & $\mathrm{F}$ & $\mathrm{M}$ & $\mathrm{F}$ & $\mathrm{M}$ & $\mathrm{F}$ & $\mathrm{M}$ & $\mathrm{F}$ & & \\
\hline Toxomerus Macquart, 1855 & 138 & 95 & 14 & 7 & 37 & 25 & 8 & 5 & 32 & 9 & 370 & 15 \\
\hline Syrphus Fabricius, 1775 & 72 & 62 & 18 & 35 & 22 & 39 & 0 & 1 & 2 & 6 & 257 & 1 \\
\hline Ocyptamus Macquart, 1834 & 13 & 69 & 2 & 24 & 3 & 47 & 6 & 27 & 1 & 28 & 220 & 24 \\
\hline Allograpta Osten Sacken, 1875 & 87 & 99 & 1 & 1 & 6 & 6 & 1 & 1 & 1 & 1 & 204 & 5 \\
\hline Microdon Meigen,1803 & 0 & 3 & 10 & 11 & 8 & 6 & 11 & 6 & 14 & 16 & 85 & 11 \\
\hline Copestylum Macquart,1846 & 2 & 4 & 0 & 7 & 1 & 7 & 1 & 18 & 0 & 5 & 45 & 10 \\
\hline Mixogaster Macquart, 1842 & 0 & 1 & 1 & 10 & 5 & 4 & 1 & 4 & 8 & 4 & 38 & 2 \\
\hline Leucopodella Hull,1948 & 1 & 6 & 0 & 7 & 0 & 3 & 0 & 2 & 0 & 10 & 29 & 2 \\
\hline Neplas Porter, 1927 & 1 & 0 & 1 & 2 & 5 & 5 & 0 & 4 & 2 & 1 & 21 & 2 \\
\hline Pseudodoros Becker, 1903 & 8 & 12 & 0 & 0 & 0 & 1 & 0 & 0 & 0 & 0 & 21 & 1 \\
\hline Aristosyrphus Curran,1941 & 0 & 0 & 0 & 5 & 0 & 7 & 0 & 4 & 0 & 1 & 17 & 3 \\
\hline Paramicrodon Meijere, 1913 & 0 & 1 & 1 & 4 & 1 & 2 & 0 & 0 & 1 & 0 & 10 & 3 \\
\hline Sphiximorpha Rondani, 1850 & 0 & 0 & 0 & 0 & 1 & 0 & 0 & 3 & 0 & 3 & 7 & 3 \\
\hline Ornidia Lepeltier \& Serville, 1828 & 83 & 0 & 0 & 0 & 1 & 1 & 0 & 0 & 0 & 0 & 5 & 1 \\
\hline Ceriogaster Williston, 1888 & 0 & 3 & 0 & 0 & 0 & 0 & 0 & 0 & 0 & 0 & 3 & 1 \\
\hline Argentinomyia L-A, 1891. & 0 & 1 & 0 & 0 & 1 & 1 & 0 & 0 & 0 & 0 & 3 & 3 \\
\hline Eumerus Meigen, 1822 & 0 & 0 & 0 & 0 & 1 & 0 & 0 & 0 & 0 & 0 & 1 & 1 \\
\hline Meromacrus Rondani, 1848 & 0 & 0 & 0 & 0 & 0 & 1 & 0 & 0 & 0 & 0 & 1 & 1 \\
\hline Myolepta Newman, 1838 & 0 & 0 & 0 & 0 & 1 & 0 & 0 & 0 & 0 & 0 & 1 & 1 \\
\hline Nausigaster Williston, 1883 & 0 & 1 & 0 & 0 & 0 & 0 & 0 & 0 & 0 & 0 & 1 & 1 \\
\hline Palpada Macquart, 1834 & 0 & 0 & 0 & 0 & 0 & 0 & 0 & 0 & 0 & 1 & 1 & 1 \\
\hline Polybiomya Shannon, 1925 & 0 & 0 & 0 & 0 & 0 & 1 & 0 & 0 & 0 & 0 & 1 & 1 \\
\hline Quichuana Knab, 1913 & 1 & 0 & 0 & 0 & 0 & 0 & 0 & 0 & 0 & 0 & 1 & 1 \\
\hline Spilomyia Meigen 1803 & 0 & 0 & 0 & 0 & 1 & 0 & 0 & 0 & 0 & 0 & 1 & 1 \\
\hline Sterphus Philippi, 1865 & 0 & 0 & 0 & 1 & 0 & 0 & 0 & 0 & 0 & 0 & 1 & 1 \\
\hline Trichopsomya Williston, 1888 & 0 & 1 & 0 & 0 & 0 & 0 & 0 & 0 & 0 & 0 & 1 & 1 \\
\hline Total & 326 & 358 & 48 & 114 & 94 & 156 & 28 & 75 & 61 & 85 & 1345 & 97 \\
\hline
\end{tabular}


1; 13 espécies em 51 indivíduos na Fase 2 e 12 espécies em 90 indivíduos na Fase 3. A menor abundância de Syrphinae foi encontrada na Fase 2, onde representou a metade do total de Syrphidae capturado (50\%).

Embora Syrphinae tenha sido a subfamília com maior abundância, foram identificados somente sete gêneros (Tabs. I, V). Os gêneros em ordem decrescente de riqueza são: Ocyptamus, com 24 espécies em 220 exemplares; Toxomerus, com 15 espécies em 370, Allograpta, com cinco espécies em 204, Argentinomyia, com três espécies em três, Leucopodella, duas espécies em 29; Syrphus, uma espécie em 257 e Pseudodoros, uma espécie em 21.

Microdontinae representou $11 \%$ do total de indivíduos coletados (Tabs. II, IV). Foram identificados quatro gêneros desta subfamília: Microdon (o mais rico, com 11 espécies em 85 exemplares); seguido por Aristosyrphus, com três espécies em 17 exemplares; Mixogaster, com duas espécies em 38 indivíduos; e Paramicrodon, com três espécies em 10 (Tab. V).

De Eristalinae, foi coletado o menor número de exemplares (7\%) (Tabs. III, IV). Entretanto, foi a subfamília que apresentou o maior número de gêneros (15): Copestylum, com 10 espécies em 45 exemplares; Sphiximorpha (3; 7); Neplas $(2 ; 21) ;$ Ornidia $(1 ; 5)$; Ceriogaster (1;3) e Eumerus, Meromacrus, Myolepta; Nausigaster, Polybiomya, Quichuana, Spilomya, Sterphus, Trichopsomya e Palpada, com uma espécie em um indivíduo coletado.

As espécies mais abundantes foram $S$. phaeostigma, com 257 espécimes capturados, $A$. neotropica com 162 e T. procrastinatus com 117 (Tab. I). As três espécies foram mais abundantes na Borda.
Syrphus phaeostigma foi dominante em três áreas: Borda (20\%), Araucária (33\%) e Fase 1 (24\%); T. procrastinatus está entre as espécies dominantes da borda (9\%), Fase 1 (12\%) e Fase 3 (13\%), enquanto que A. neotropica foi dominante somente na Borda (22\%).

Novos registros de Syrphidae. Das 97 espécies reconhecidas, 61 foram identificadas e 36 foram associadas a gêneros. Destas, acredita-se que grande parte seja de espécies novas, porém, existe uma grande dificuldade na identificação devido à carência de chaves adequadas para as espécies neotropicais havendo a necessidade de um estudo taxonômico mais aprofundado.

Até o momento, foram identificadas como novas uma espécie de Microdon, uma de Paramicrodon e uma de Aristosyrphus, sendo que esta última está em fase de publicação e as outras duas serão descritas oportunamente.

Análise faunística. Através da análise de agrupamento das cinco áreas amostradas com dados de presença das espécies pelo índice de Jaccard, observouse uma maior semelhança entre as Fase 2 e Fase 3, as mais preservadas, e entre as áreas de Araucária e Fase 1. A Borda ficou isolada das demais (Fig. 1).

Das 97 espécies identificadas, somente sete foram comuns às cinco áreas: Copestylum selectum (Curran, 1939), Leucopodella gracilis, Mixogaster polistes Hull, 1954, Ocyptamus funebris Macquart, 1834, S. phaeostigma, T. procrastinatus e T. tibicen (Wiedemann, 1830). A área com maior número de espécies exclusivas foi a de Borda (28), seguida pela Fase 1 (11), Araucária e Fase 3 (quatro) e Fase 2 (três) (Fig. 2). O maior número de espécies comuns ocorreu entre a área de Araucária e Fase 1 (25) e o menor entre as áreas de Borda com as Fases 2 e 3 (10).

Tabela VI. Abundância e riqueza de Syrphidae por guildas tróficas nas cinco áreas inventariadas no Parque Estadual Vila Velha, Ponta Grossa, Paraná, setembro de 1999 a agosto de 2000.

\begin{tabular}{llllllrr}
\hline Guildas tróficas & \multicolumn{3}{c}{ Áreas } & Total & Espécies \\
\cline { 2 - 5 } & \multicolumn{1}{c}{ Borda } & Araucária & Fase 1 & Fase 2 & Fase 3 & & \\
Saprófagos & $10(1,5 \%)$ & $8(5 \%)$ & $12(4,8 \%)$ & $19(18,4 \%)$ & $6(4,1 \%)$ & 55 & 18 \\
Fitófagos & $1(0,1 \%)$ & $3(1,9 \%)$ & $11(4,4 \%)$ & $4(3,9 \%)$ & $3(2,1 \%)$ & 22 & 1 \\
Mirmecófagos & $5(0,7 \%)$ & $42(26 \%)$ & $33(13,2 \%)$ & $26(25,2)$ & $44(30,1 \%)$ & 150 & 19 \\
Predadores & $663(97 \%)$ & $109(67 \%)$ & $191(76,4 \%)$ & $51(49,5 \%)$ & $90(61,6 \%)$ & 1.104 & 51 \\
Não identificadas & $5(0,7 \%)$ & 0 & $3(1,2 \%)$ & $3(2,9 \%)$ & $3(2,1 \%)$ & 14 & 8 \\
Total & 684 & 162 & 250 & 103 & 146 & 1.345 & 97 \\
\hline
\end{tabular}

Tabela VII. Número de espécies de sirfídeos capturados por armadilha Malaise nas cinco áreas e valores estimados de riqueza pelos métodos de Chao 1, Chao 2, Jack-Knife 1 (Jack1), Jack-knife 2 (Jack2), Bootstrap e Michaelis-Menten (MM).

\begin{tabular}{|c|c|c|c|c|c|c|c|c|c|}
\hline \multirow[t]{2}{*}{ Áreas } & \multirow[t]{2}{*}{ Espécies } & \multirow[b]{2}{*}{ Singletons } & \multirow[b]{2}{*}{ Doubletons } & \multicolumn{6}{|c|}{ Estimadores } \\
\hline & & & & Chao 1 & Chao 2 & Jack1 & Jack2 & Bootstrap & MM \\
\hline Borda & 54 & $25(46 \%)$ & $6(11 \%)$ & $106 \pm 31$ & $106 \pm 31$ & $79 \pm 6$ & 97 & 64 & 65 \\
\hline Araucária & 34 & $11(32 \%)$ & $4(12 \%)$ & $49 \pm 12$ & $55 \pm 16$ & $47 \pm 4$ & 56 & 40 & 59 \\
\hline Fase 1 & 51 & $23(45 \%)$ & $8(16 \%)$ & $84 \pm 19$ & $90 \pm 22$ & $76 \pm 5$ & 92 & 61 & 78 \\
\hline Fase 2 & 27 & $9(33 \%)$ & $7(26 \%)$ & $33 \pm 5$ & $35 \pm 7$ & $37 \pm 3$ & 41 & 32 & 36 \\
\hline Fase 3 & 31 & $12(39 \%)$ & $6(19 \%)$ & $43 \pm 9$ & $43 \pm 9$ & $43 \pm 4$ & 49 & 36 & 45 \\
\hline
\end{tabular}

Tabela VIII. Proporção sexual das três espécies mais abundantes do total de sirfídeos coletados no período de setembro de 1999 a agosto de 2000, nas cinco áreas inventariadas do Parque Estadual Vila Velha, Ponta Grossa, PR.

\begin{tabular}{lcccrr}
\hline Espécies & Borda & Araucária & Fase 1 & Fase 2 & Fase 3 \\
\hline Syrphus phaeostigma & F/M & F/M & F/M & F/M & F/M \\
Allograpta neotropica & $0,9: 1$ & $1,9: 1$ & $1,8: 1$ & $1: 0$ & $3: 1$ \\
Toxomerus procrastinatus & $1: 1$ & $0: 1$ & $1,3: 1$ & $1: 1$ & 0 \\
Total & $0,8: 1$ & $0,2: 1$ & $0,6: 1$ & $0: 2$ & $0,1: 1$ \\
\hline
\end{tabular}


Guildas tróficas. Dos quatro grupos de guildas tróficas reconhecidos nas cinco áreas, houve predominância dos predadores (51 espécies; abundância $82 \%$ ), sendo todos da subfamília Syrphinae, seguidos pelos mirmecófagos $(19 ; 11 \%)$ da subfamília Microdontinae, saprófagos (18; $4 \%$ ) e fitófagos (1;2\%), ambos Eristalinae. Oito espécies não tiveram suas guildas tróficas identificadas, representando $1 \%$ do total (Tab. VI). A área com maior proporção de predadores foi a Borda ( $97 \%$ das espécies), seguida, em ordem decrescente, pela Fase $1(76,4 \%)$, Araucária (67\%), Fase 3 (61,6\%) e Fase 2 (49,5\%) (Fig. 3).

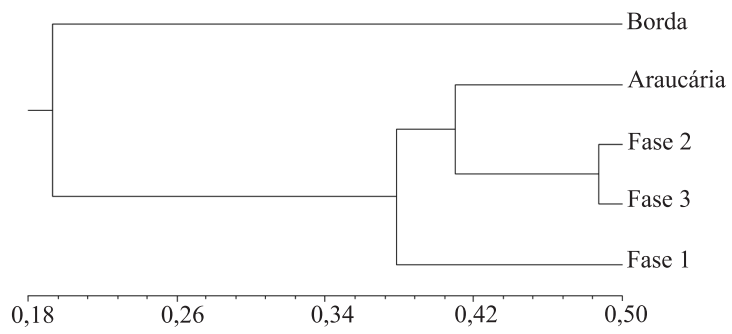

Fig. 1. Análise de agrupamento pelo Índice de Jaccard, a partir de dados de presença e ausência de espécies de Syrphidae, capturados com armadilhas Malaise nas cinco áreas amostradas no período de setembro de 1999 a agosto de 2000 no Parque Estadual de Vila Velha, Ponta Grossa, Paraná.

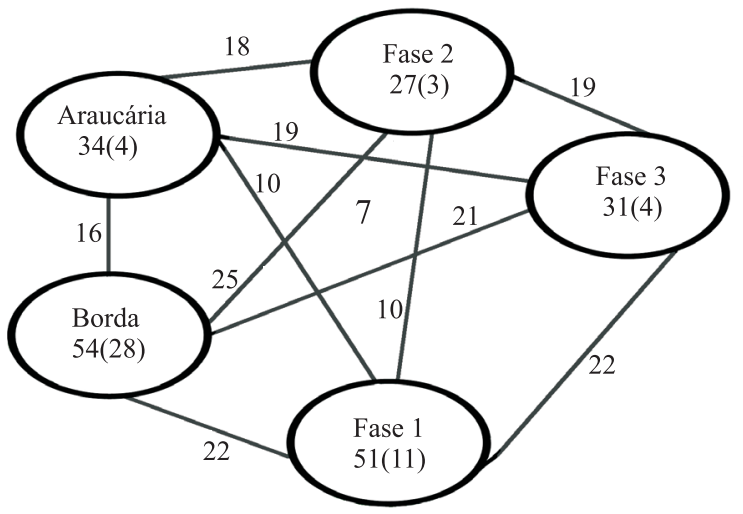

Fig. 2. Comparação entre as cinco áreas amostradas no período de setembro de 1999 a agosto de 2000 no Parque Estadual de Vila Velha, Ponta Grossa, Paraná, indicando o número de espécies encontradas em cada área, número de espécies exclusivas de cada área (entre parênteses), número de espécies comuns entre cada área e ao centro o número de espécies comuns a todas as áreas.

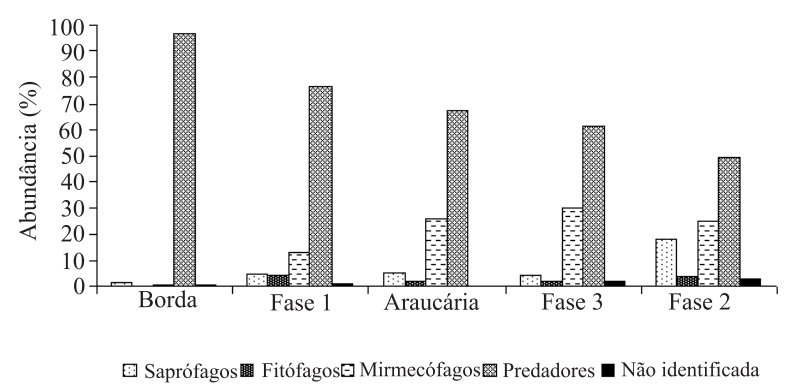

Fig. 3. Abundância percentual das guildas tróficas de Syrphidae capturadas com armadilhas Malaise nas cinco áreas do Parque Estadual Vila Velha, Ponta Grossa, Paraná durante o período de setembro de 1999 a agosto de 2000.
Na Fase 2, onde foi registrada a menor proporção de predadores (50\%), $25 \%$ foram mirmecófagos e $25 \%$ saprófagos e fitófagos.

Estimadores de riqueza. Utilizando-se os valores semanais de captura obteve-se, através dos métodos Chao 1, Chao 2, Jack-Knife 1, Jack-Knife 2, Bootstrap e Michaelis-Menten, o valor de riqueza estimada para cada área (Tab. VII). Dentre os métodos, o Chao 1 e Chao 2 foram os que resultaram numa estimativa de riqueza de espécies maior na Borda e Michaelis-Menten na área de Araucária, enquanto que nas áreas em Fase de sucessão vegetal (Fase 1, Fase 2 e Fase 3) foi o Jack-Knife 2. Já o método Bootstrap resultou nos menores valores estimados em todas as áreas, sendo os mais próximos dos que foram efetivamente capturados.

A área com maior proporção de espécies "singletons" foi a Borda, com $46 \%$ do total de espécies capturado para área (25 espécies). A menor proporção ocorreu na área de Araucária, com 32\% do total (11). A maior proporção de espécies "doubletons" ocorreu na Fase 2 com $26 \%$ do total capturado para a área (sete) e a menor proporção ocorreu na Borda, com $11 \%$ (seis) (Tab. VII).

$\mathrm{Na}$ Borda, os estimadores Chao 1 e Chao 2 indicaram que aproximadamente $50 \%$ das espécies teriam sido coletadas e conforme o Bootstrap, $84 \%$ das espécies. $\mathrm{Na}$ área de Araucária, a estimativa pelo Chao 2 foi de que $69 \%$ das espécies teriam sido coletadas e pelo Bootstrap, $85 \%$. Na Fase 1, houve uma estimativa de que 57\% das espécies teriam sido capturadas pelo Chao 2 e pelo Bootstrap, $84 \%$. Na Fase 2, a estimativa pelo Chao 2 foi de $77 \%$ e pelo Bootstrap, 32 (97\%). Na Fase 3, 72\% pelo Chao e pelo Bootstrap, $86 \%$.

Proporção sexual. De forma geral, nas cinco áreas amostradas foram capturadas mais fêmeas do que machos. Porém, entre as três espécies que foram mais abundantes do total de sirfídeos coletados, observou-se a seguinte proporção de fêmeas para machos: Syrphus phaeostigma: Borda (0,9: 1), Araucária (1,9: 1), Fase 1 (1,8: 1), Fase 2 (1: $0)$ e Fase 3 (3: 1); Allograpta neotropica: Borda (1: 1), Araucária (0:1), Fase 1 (1,3:1) e Fase 2 (1: 1); Toxomerus procrastinatus: Borda (0,8: 1), Araucária (0,2: 1), Fase 1 $(0,6: 1)$, Fase $2(0: 2)$ e Fase $3(0,1: 1)$ (Tab. VII).

\section{DISCUSSÃO}

A menor abundância de dípteros ocorreu na Borda e a maior na área de Araucária, área com vegetação mais densa e com menor penetração de luz. A elevada abundância de Diptera em áreas de interior de floresta pode estar relacionada com a maior presença de matéria orgânica em decomposição, além de fungos, que são utilizados como alimento por diversos grupos de imaturos pertencentes a essa Ordem (STORK, 1991; DidHAM, 1997).

Ganho \& Marinoni (2003), estudando os coleópteros do primeiro ano do PROVIVE, também verificaram uma menor abundância na Borda, o que foi atribuído por esses autores à menor disponibilidade de nichos para as espécies capturadas com Malaise (devido provavelmente à estrutura da vegetação da área, que é mais aberta com predominância de plantas arbustivas).

Ao contrário de todos os dípteros, a maior abundância de Syrphidae foi encontrada na Borda, área 
de transição, com grande luminosidade. Foi também observada nessa área a maior riqueza de espécies da família. Essas constatações devem-se, provavelmente, ao fato dos sirfídeos serem mais relacionados com regiões abertas e iluminadas, em áreas com vegetais em floração (uma vez que na fase adulta alimentam-se de néctar e pólen de flores) (Vockeroth \& THOMPSON, 1987; Owen, 1991; MARINONi et al., 2004). Há também, na Borda, maior disponibilidade de nichos; possibilitando uma maior oferta de recursos alimentares tanto às larvas quanto aos sirfídeos adultos. Observa-se também que o maior número de exemplares (assim como o maior número de espécies de Syrphidae) foram encontrados nas áreas de maior interferência antrópica (Borda e Fase 1), o que indica relação com os estágios de sucessão das áreas.

Nas outras áreas, as maiores riquezas e abundância de Syrphidae apresentaram relação com a luminosidade e com a distância da Borda. A Fase 1 apresentou maior riqueza e abundância do que a área de Araucária, apesar dessa última estar mais próxima da Borda. Porém, na Fase 1, a vegetação em estágio inicial a intermediário de sucessão vegetal apresenta dossel mais aberto e maior entrada de luz do que a área de Araucária. Isso também foi verificado nas Fases 2 e 3, ambas apresentando estágio de sucessão vegetal variando de intermediário a avançado, sendo a Fase 2 a com menor luminosidade, devido ao dossel mais fechado. Esta última, embora mais próxima da Borda, apresentou menor riqueza e abundância do que a Fase 3.

GILBERT (1986) reconheceu quatro grupos: larvas que se alimentam de tecidos de plantas (fitófagas), que se alimentam de matéria orgânica em decomposição (saprófagas), que vivem em ninhos de insetos sociais (mirmecófagas) e as carnívoras predadoras, que se alimentam principalmente de afídeos.

Quando a abundância observada é avaliada considerando essas guildas, há uma coincidência dos resultados obtidos com os grupos taxonômicos avaliados, principalmente quanto às espécies pertencentes a Syrphinae.

Com o avanço do processo de sucessão vegetal nas áreas, diminuiu a proporção de predadores e aumentou a proporção de mirmecófagos (Fig. 3). Observase que, nas áreas com vegetação em processo de sucessão natural (Fase 1: inicial a intermediária; Fase 2 e Fase 3: intermediária a avançada), a presença de Syrphinae diminuiu à medida que o processo de sucessão avançou. Essa diminuição de Syrphinae pode estar relacionada ao fato da grande maioria ser predadora de afídeos, na fase de imaturos e, segundo Brown (1984), plantas arbustivas características de estágios iniciais de sucessão vegetal apresentam maior número de afídeos do que em estágios mais avançados. A ocorrência de maior número de predadores na Borda já era esperada, uma vez que essa área apresenta predominância de gramíneas e herbáceas com flores que servem como fontes de alimentos aos sirfídeos adultos (néctar e pólen) e para as larvas (afideos). Além disso, apresenta insolação direta e espaço mais aberto para o vôo.

Na Fase 2, observou-se uma diminuição do número de predadores e um aumento das outras guildas. Nessa área, a subfamília mais abundante foi Eristalinae, na qual são encontrados representantes com hábitos alimentares variados na fase de imaturos, podendo ser saprófagos e fitófagos (STAHLs et al., 2003). Desta forma, as condições vegetais da área (vegetação mais alta, dossel mais fechado, menor entrada de luz e maior umidade), poderiam disponibilizar maior oferta alimentar para o estágio imaturo desses sirfídeos.

A maior proporção de mirmecófagos ocorreu na Fase 3, a qual se caracteriza como uma área florestada, sendo a mais preservada entre todas. As espécies caracterizadas nessa guilda, que já tiveram algum estudo realizado, estão alocadas em Microdontinae. As formas imaturas de Microdontinae, segundo a literatura disponível, são mirmecófagas, vivendo associadas a ninhos de formigas. As fêmeas adultas ovipositam nos ninhos e os imaturos provavelmente se alimentam das formas jovens (Thompson, 1981a; DufFIELD, 1981; Marinoni \& Bonatto, 2002; Stahls et al., 2003).

De acordo com os estimadores de riqueza, observou-se que nas duas áreas com níveis mais avançados de sucessão vegetal e mais preservadas (Fase 2 e Fase 3), os valores estimados foram mais próximos dos valores de captura (variando entre $72 \%$ e $97 \%$ ), para a maioria das espécies coletadas. Entretanto, nas áreas em níveis de sucessão vegetal de inicial a intermediário (Fase 1) - área de Araucária e Borda - a diferença entre os valores estimados e os que foram efetivamente obtidos foi maior, variando entre $50 \%$ a $85 \%$. Esses dados poderiam indicar a existência de um número maior de espécies a serem coletadas nesses locais. Isso se torna mais evidente quando se observa o gráfico com a curva de acumulação de espécies da Borda, que mostra um crescente aumento de espécies até o final das coletas, não atingindo assíntota (enquanto que nas áreas Fase 2 e Fase 3 a curva estabilizou em torno da $20^{a}$ semana de coleta).

$\mathrm{Na}$ análise de agrupamento realizada entre as áreas, aquelas em fase de sucessão mais avançada (Fases 2 e 3 ) foram as que mais se aproximaram, sendo a Borda a mais afastada, indicando uma estruturação da fauna de Syrphidae diferenciada entre as áreas. Verificou-se alto percentual de espécies exclusivas na Borda (52\%) e diminuição do percentual nas áreas de interior de floresta, sendo em ordem decrescente: Fase 1 (21\%), Fase $3(13 \%)$, Araucária (12\%) e Fase $2(11 \%)$. Essa tendência foi verificada também em estudos realizados com Coleoptera nas mesmas áreas (GANHO \& MARINONI, 2003).

Ocyptamus, Toxomerus, Microdon e Copestylum (os gêneros mais ricos em espécies neste estudo) estão descritos na literatura como os que possuem maior número de espécies na região Neotropical (THOMPSON, 1981b, 1999; MARINONI et al., 2004).

Com relação à proporção sexual, embora o número de fêmeas tenha sido maior do que o número de machos nas cinco áreas, na Fase 2 observou-se uma proporção bem maior do que nas outras. Essa área apresenta vegetação mais alta, dossel mais fechado e espaços mais sombreados. Esse fato corrobora o estudo realizado por GiLBert (1985), no qual foi observado que machos de todas as espécies voam igualmente, tanto no sol quanto na sombra, mas fêmeas voam mais em locais sem sol direto. Outrossim, segundo OwEN (1991), as fêmeas podem ser 
capturadas em maior número por serem mais ativas que os machos procurando locais para oviposição.

Agradecimentos. Agradecemos a Norma G. Ganho, por disponibilizar o material coletado durante o primeiro ano do PROVIVE. A Sionei Ricardo Bonatto, pela concessão do programa gerenciador de biodiversidade (BONABio). Ao Conselho Nacional de Desenvolvimento Científico e Tecnológico (CNPq) e à Coordenação de Aperfeiçoamento de Pessoal de Nível Superior (CAPES), pelas bolsas concedidas.

\section{REFERÊNCIAS BIBLIOGRÁFICAS}

Brown, V. K. 1984. Secondary Succession: Insect-Plant Relationships. Bioscience 34(11):710-716.

Cowell, R. K. 2000. Estimates: Statistical estimation of species richness and shared species from samples. Version 6.0b1. On-line User's Guide Draft 20 september 2000. Disponível em: <http://viceroy.eeb.uconn.edu/ estimates>. Acesso em: 10.2004.

Didham, R. K. 1997. Dipteran tree-crown assemblages in a diverse southern temperate rain forest. In: STORK, N. E.; AdIS, J. \& Didham, R. K. eds. Canopy Arthropods. London, Chapman \& Hall. p.320-343.

DufFIELD, R. M. 1981. Biology of Microdon fuscipennis (Diptera: Syrphidae) with interpretations of the reproductive strategies of Microdon species found North of Mexico. Proceedings of the Entomological Society of Washington 83(4):716-724.

Ganho, N. G. \& Marinoni, R. C. 2003. Fauna de Coleoptera no Parque Estadual de Vila Velha, Ponta Grossa, Paraná, Brasil. Abundância e riqueza das famílias capturadas através de armadilhas Malaise. Revista Brasileira de Zoologia 20(4):727-736.

Gilbert, F. S. 1985. Diurnal activity patterns in hoverflies (Diptera, Syrphidae). Ecological Entomology 10:385-392. 1986. Hoverflies. London, Cambridge University. 67p.

Harbach, R. E. 1984. A new species of Toxomerus (Diptera, Syrphidae) from Brazil, with notes on three related species. Proceedings of the Entomological Society of Washington 86(4):840-844.

Hippa, H. \& Thompson, F. C. 1983. Meropidia, a new genus of flower flies (Diptera, Syrphidae) from South America. Papéis Avulsos de Zoologia 35(9):109-115.

Humphrey, J. W.; Hawes, C.; Peace, A. J.; Ferris-Kaan, R. \& Jukes, M. R. 1998. Relationships between insect diversity and habitat characteristics in plantation forest. Forest Ecology and Management 113(1):11-21

LAwton, J. H. 1983. Plant architecture and the diversity of phytophagous insects. Annual Review of Entomology 28:23-39

Marinoni, L. \& Bonatto, S. R. 2002. Sazonalidade de três espécies de Syrphidae (Insecta, Diptera) capturadas com armadilha Malaise no Estado do Paraná, Brasil. Revista Brasileira de Zoologia 19(1):95-104.

Marinoni, R. C. \& Dutra, R. R. C. 1993. Levantamento da Fauna Entomológica no Estado do Paraná. I. Introdução. Situações climáticas e florística de oito pontos de coleta. Dados faunísticos de agosto de 1986 a julho de 1987. Revista Brasileira de Zoologia 8(1/2/3/4):31-73.

Marinoni, R. C \& Ganho, N. G. 2003. Fauna de Coleoptera no Parque Estadual de Vila Velha, Ponta Grossa, Paraná, Brasil. Abundância e riqueza das famílias capturadas através de armadilhas Malaise. Revista Brasileira de Zoologia 20(4):737-744.

Marinoni, L.; Miranda, G. F. \& Thompson, F. C. 2004. Abundância e riqueza de espécies de Syrphidae (Diptera) em áreas de borda e interior de floresta no Parque Estadual de Vila Velha, Ponta Grossa, Paraná, Brasil. Revista Brasileira de Entomologia 48(4):553-559.

Novotny, V. \& BASSET, Y. 2000. Rare species in communities of tropical insect herbivores: pondering the mystery of singletons. Oikos 89:564-572

Owen, J. 1991. The ecology of a garden: the first fifteen years. Cambridge, Cambridge University. 403p.

Rohlf, F. J. 1989. NTSYS-PC. Numerical Taxonomy and Multivariate Analysis System. New York, Exeter. vi+187p

Sneath, P. H. \& Sokal, A. R. R. 1973. Numerical taxonomy. San Francisco, Freeman. 573p.

Southwood, T. R. E.; Brown, V. K. \& Reader, P. M. 1979. The relationships of plant and insect diversities in succession. Biological Journal of the Linnean Society 12:327-348.

Stahls, G.; Hippa, H.; Rotheray, G.; Muona, J. \& Gilbert, F. 2003. Phylogeny of Syrphidae (Diptera) inferred from combined analysis of molecular and morphological characters. Systematic Entomology 28(4):433-450.

Stork, N. E. 1991. The composition of the arthropod fauna of Bornean low land rain forest trees. Journal of Tropical Ecology 7:161-180.

Thompson, F. C. 1969. A new genus of Microdontinae flies (Diptera: Syrphidae) with notes on the placement of the subfamily. Psyche 76(1):74-85

1981a. Revisionary notes on Neartic Microdon flies (Diptera: Syrphidae). Proceedings of the Entomological Society of Washington 83(4):725-758.

1981b. The flower flies of the West Indies (Diptera: Syrphidae). Memorials of the Entomological Society of Washington 9:1-200.

1991. The flower fly genus Ornidia (Diptera, Syrphidae). Proceedings of the Entomological Society of Washington 93(2):248-261.

1997a. Revision of the Eristalis flower flies (Diptera: Syrphidae) of the Americas South of the United States. Proceedings of the Entomological Society of Washington 99(2):209-237.

1997b. Spilomyia flower flies of the New World (Diptera: Syrphidae). Memorials of the Entomological Society of Washington 18:261-272.

1999. A key to the genera of the flower flies of the Neotropical Region including descriptions of new genera and new species and a glossary of taxonomic terms. Contributions on Entomology International 3(3):322-378.

Vockeroth, J. R \& Thompson, F. C. 1987. Syrphidae. In: McAlpine, J. F. ed. Manual of Neartic Diptera Otawa, Agriculture Canada Research. v. 2, p.713-743. 